\title{
Effect of the Great East Japan Earthquake on Cardiovascular Diseases
}

Tatsuo Aoki, MD, PhD; Jun Takahashi, MD, PhD; Yoshihiro Fukumoto, MD, PhD; Satoshi Yasuda, $\mathrm{MD}, \mathrm{PhD}$; Kenta Ito, $\mathrm{MD}, \mathrm{PhD}$; Satoshi Miyata, $\mathrm{PhD}$;

Tsuyoshi Shinozaki, MD, PhD; Kanichi Inoue, MD, PhD; Tetsuo Yagi, MD, PhD;

Tatsuya Komaru, MD, PhD; Yoshiaki Katahira, MD, PhD; Atsushi Obata, MD;

Tetsuya Hiramoto, MD, PhD; Hiroyasu Sukegawa, MD, PhD;

Kazunori Ogata, MD, PhD; Hiroaki Shimokawa, MD, PhD

\begin{abstract}
Background: We reported an increased occurrence of cardiovascular diseases (CVDs) after the Great East Japan Earthquake by examining ambulance records, but it had to be confirmed by cardiologists.

Methods and Results: We enrolled patients admitted to the cardiology department of the 10 hospitals in the disaster area from 4 weeks prior to 15 weeks after March 11 in the years 2008-2011 $(n=14,078)$. The weekly occurrence of several CVDs, including heart failure (HF), pulmonary thromboembolism (PTE) and infectious endocarditis (IE), was sharply and significantly increased after the Earthquake.
\end{abstract}

Conclusions: The Disaster caused significantly increases in the occurrence of HF, PTE and IE. (Circ J 2013; 77: 490-493)

Key Words: Cardiovascular disease; Disasters; Great East Japan Earthquake

W e examined ambulance records from Miyagi prefecture and reported that the occurrence of cardiovascular diseases (CVDs), including heart failure (HF), acute coronary syndrome (ACS), stroke, and cardiopulmonary arrest, had increased after the Great East Japan Earthquake (magnitude 9.0 on March 11, 2011). ${ }^{1}$ However, because the ambulance records were made in the emergency rooms by doctors who were not always cardiologists, our findings had to be confirmed by cardiologists in the disaster area. Furthermore, we did not examine the incidence of pulmonary thromboembolism (PTE), infectious endocarditis (IE) or takotsubo cardiomyopathy in that previous study because those diagnoses require a professional approach. ${ }^{1}$

In this study, we examined the medical records made by cardiologists to determine whether the occurrence of CVDs, including HF, acute myocardial infarction (AMI), PTE, IE and takotsubo cardiomyopathy, had increased after the Earthquake.

\section{Methods}

The ethical committees of Tohoku University Hospital and participating hospitals approved the protocol of the present study.

\section{Study Population and Participating Hospitals}

We enrolled all patients admitted to the cardiology department of the 10 hospitals in Miyagi prefecture from 4 weeks prior to 15 weeks after the Earthquake in 2011 and in the corresponding periods in 2008, 2009 and $2010(\mathrm{n}=14,078)$. We also col-

Received December 24, 2012; revised manuscript received January 5, 2013; accepted January 7, 2013; released online January 18, 2013 Time for primary review: 3 days

Department of Cardiovascular Medicine, Tohoku University Graduate School of Medicine, Sendai (T.A., J.T., Y.F., K. Ito, S.M., H. Shimokawa); Department of Cardiovascular Medicine, National Cerebral and Cardiovascular Center Hospital, Suita (S.Y.); Department of Cardiovascular Medicine, Sendai Medical Center, Sendai (T.S.); Department of Cardiovascular Medicine, South Miyagi Medical Center, Okawara (K. Inoue); Department of Cardiovascular Medicine, Sendai City Hospital, Sendai (T.Y.); Division of Cardiology, Tohoku Rosai Hospital, Sendai (T.K.); Cardiovascular Center, Tohoku Kouseinenkin Hospital, Sendai (Y.K.); Department of Cardiovascular Medicine, Saka General Hospital, Shiogama (A.O.); Department of Cardiovascular Medicine, Osaki Citizen Hospital, Osaki (T.H.); Department of Cardiovascular Medicine, Japanese Red Cross Ishinomaki Hospital, Ishinomaki (H. Sukegawa); and Department of Cardiovascular Medicine, Kesennuma City Hospital, Kesennuma (K.O.), Japan

The Guest Editor for this article was Hiroshi Ito, MD.

Mailing address: Hiroaki Shimokawa, MD, PhD, Professor and Chairman, Department of Cardiovascular Medicine, Tohoku University Graduate School of Medicine, Seiryo-machi, Aoba-ku, Sendai 980-8575, Japan. E-mail: shimo@ cardio.med.tohoku.ac.jp

ISSN-1346-9843 doi:10.1253/circj.CJ-12-1594

All rights are reserved to the Japanese Circulation Society. For permissions, please e-mail: cj@j-circ.or.jp 
A.

HF

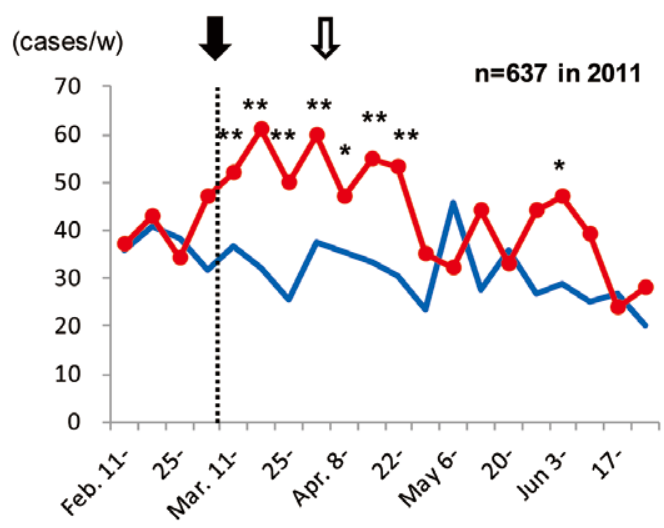

c.

PTE

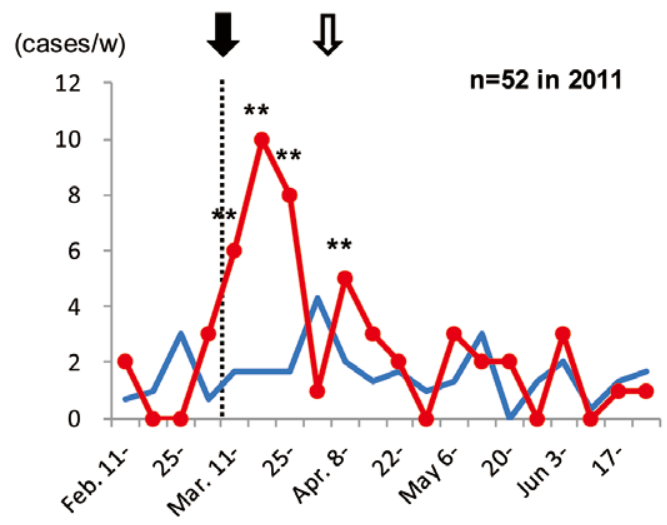

\section{E. Takotsubo Cardiomyopathy}

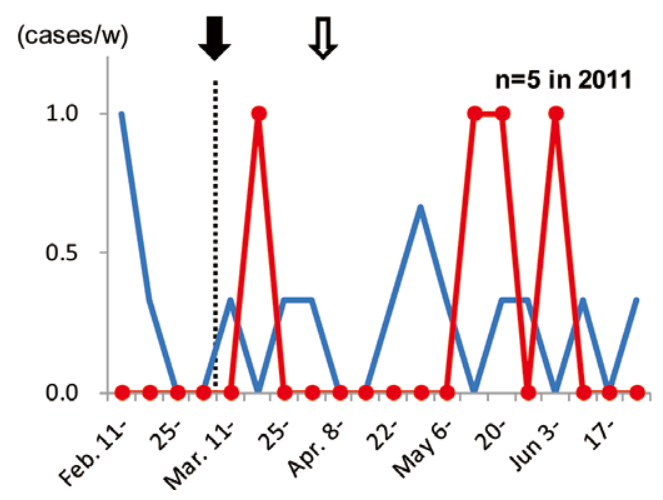

B. AMI

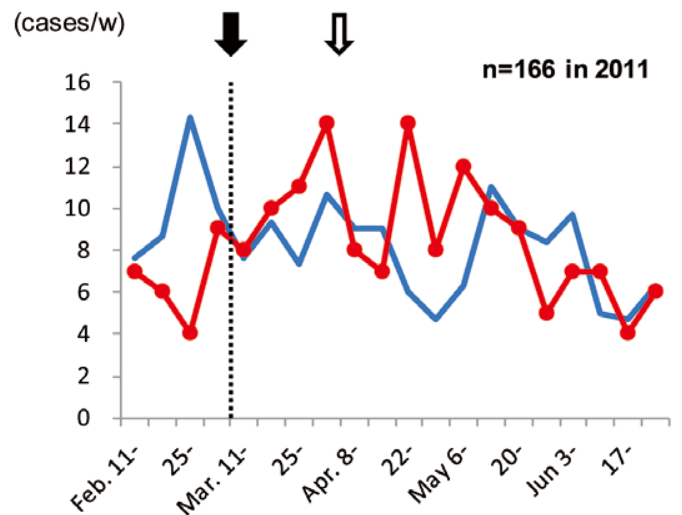

D.

IE

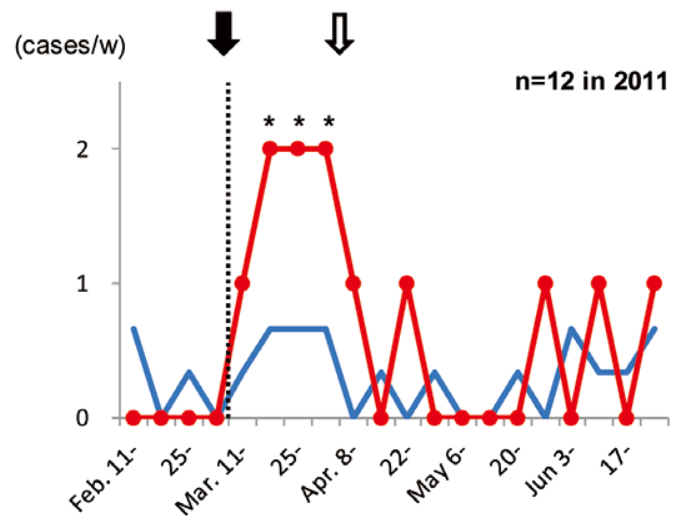

Figure 1. Weekly occurrence of cardiovascular diseases: (A) heart failure (HF), (B) acute myocardial infarction, (C) pulmonary thromboembolism (PTE), (D) infectious endocarditis (IE) and (E) takotsubo cardiomyopathy. HF, PTE and IE were significantly increased after the Earthquake. ${ }^{*} \mathrm{P}<0.05$, ${ }^{\star *} \mathrm{P}<0.01$. Black arrows indicate the occurrence of the Great East Japan Earthquake (magnitude 9.0, March 11, 2011), and white arrows indicate the largest aftershock (magnitude 7.2, April 7, 2011).

lected additional information about the date of admission, sex and age of the patients from the medical insurance database. We defined the 3 hospitals facing the Pacific Ocean as those in the seacoast area with direct assault by the tsunamis, and the remaining 7 hospitals as those in the inland (remote) area.

\section{Definition of the Diseases}

All definitive diagnoses of the patients were confirmed at discharge by cardiologists and classified according to the International Statistical Classification of Diseases and Related Health Problems, 10th revision (ICD-10). We also collected the diag- 


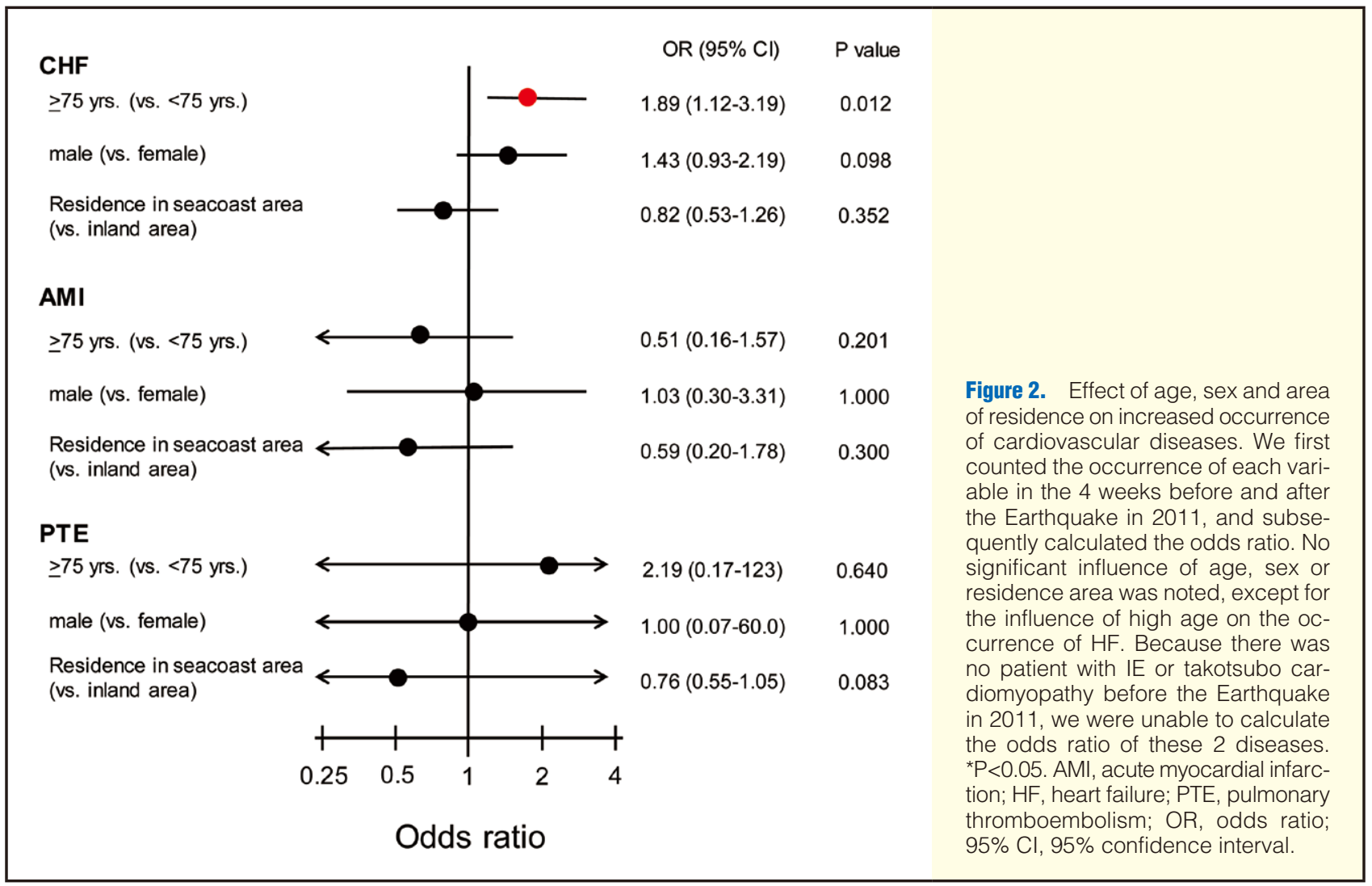

nosis at discharge from the medical insurance database as the ICD-10 code, comprising I-50.0 (HF), I-21.0-I-21.9 (AMI), I-26.0-I-26.9 (PTE), I-33.0-I-33.9 (IE) and takotsubo cardiomyopathy (I-51.8).

\section{Statistical Analysis}

We used a Poisson regression model to assess differences in the variables between 2011 and the previous 3 years. ${ }^{1}$ Furthermore, as previously reported, ${ }^{1}$ we calculated the odds ratio with the 4-week occurrence in 2011 before and after the Earthquake in terms of age ( $<75$ or $\geq 75$ years), sex, and area of residence (inland vs. seacoast). Continuous variables are expressed as mean \pm SD. All statistical analyses were performed using $\mathrm{R}$ 2.15.0 (www.r-project.org/). All P values were 2-sided, and $\mathrm{P}<0.05$ was considered to be statistically significant.

\section{Results}

The number of patients enrolled in the study for 2008, 2009, 2010 and 2011 was 3,190, 3,582, 3,752 and 3,554, respectively. In 2011, the prevalence of male sex was significantly lower $(62.2 \%, 61.75,59.95$ and $58.8 \%$ in 2008, 2009, 2010 and 2011, respectively, $\mathrm{P}=0.014$ ) and age (years) was significantly higher $(68.8 \pm 13.9,69.5 \pm 13.9,70.4 \pm 14.2$, and $71.2 \pm 14.2$ in 2008, 2009, 2010 and 2011, respectively, $\mathrm{P}<0.05$ ).

The weekly occurrence of each of HF, PTE and IE was significantly increased after the Earthquake (Figures 1A,C,D). We also noted a mild but insignificant peak of the weekly occurrence of AMI after the Earthquake (Figure 1B). There were very few cases of takotsubo cardiomyopathy, even after the Earthquake (Figure 1E). The significant increase in the weekly occurrence of HF was prolonged for 7 weeks after the Earth- quake in 2011 (Figure 1A), whereas the time course of PTE showed a second peak at the largest aftershock (magnitude 7.2 on April 7, 2011).

The subgroup analyses showed that among the 3 factors examined (age, sex, and area of residence), only higher age ( $>75$ years) significantly influenced the occurrence of HF but not that of AMI or PTE (Figure 2). Because there was no patient with IE or takotsubo cardiomyopathy for 4 weeks before the Earthquake in 2011, we were unable to calculate the odds ratio of either disease.

\section{Discussion}

In the present study of cardiologists records, as compared with our recent study using ambulance records, ${ }^{1}$ we were able to demonstrate the following: (1) a sharp and sustained (over 7 weeks) increase in the occurrence of HF after the Earthquake, (2) a sharp but transiently increased occurrence of both PTE and IE after the Earthquake, and (3) a tendency for the occurrence of AMI to be increased, but not that of takotsubo cardiomyopathy, after the Earthquake.

\section{Increased Occurrences of CVD}

The present study demonstrated a significant increase in the occurrence of both HF and PTE, consistent with the findings of our recent study ${ }^{1}$ and another study, ${ }^{2}$ and of IE, which was a novel finding not reported previously. ${ }^{3-8}$

The Earthquake forced many people in the Miyagi prefecture to take shelter and/or to live without daily necessities, services, and medicines. Disaster situations can increase the occurrence of CVDs through physical and mental stresses. ${ }^{9}$ Furthermore, a prolonged stressful situation can suppress the immune sys- 
tem, ${ }^{10}$ leading to increased rates of infectious diseases, such as IE.

Activation of the sympathetic nervous system of people involved in the present disaster would have elevated both blood pressure and heart rate, as previously reported.9,11 The report by Satoh et al has also demonstrated that self-monitored blood pressure significantly increased after the Earthquake.12 Furthermore, we recently reported that the Earthquake increased the occurrence of ventricular tachyarrhythmias among patients with an implantable cardiac defibrillator. ${ }^{13}$ Thus, we consider that an activated sympathetic nervous system, elevated blood pressure, and increased occurrence of tachyarrhythmias were all involved in the increased occurrence of HF during and after the Great East Japan Earthquake.

Although people in temporary accommodation were supplied with information and compression stockings, the increased occurrence of PTE after the Earthquake was not prevented. ${ }^{3}$ The occurrence of severe PTE, with resultant improved mortality from PTE, may have been decreased; however, further studies regarding the effects of preventive practice for PTE are needed.

In the present study, the occurrence of AMI also tended to increase after the Earthquake, and in our recent study there was a significant increase in the occurrence of ACS (AMI plus unstable angina). ${ }^{1}$ Unlike in a previous report, ${ }^{3}$ we did not observe an increased occurrence of takotsubo cardiomyopathy. The reasons for the discrepancy remains to be examined in future studies.

\section{Effects of Age, Sex and Location of Hospitals on CVDs}

In the present study, no significant influence of age, sex or area of residence was noted for CVDs, except for the influence of higher age on the occurrence of $\mathrm{HF}$, which suggested that the Earthquake had a greater effect on elderly people.

Although the tsunami directly and seriously affected the seacoast area, the increased occurrence of CVDs after the Earthquake was comparable between the seacoast and inland areas. Similar indirect effects of a disaster on CVD occurrence were reported after the World Trade Center Disaster in 2001, whereby the blood pressure of people in Mississippi was equally elevated as in those in New York City. ${ }^{14}$ These results indicate that life-threatening events, such as a great earthquake, can trigger CVDs even in remote areas.

The limitations of this study include the lack of detailed patient data, such as clinical characteristics and underlying heart disease. In order to prospectively observe the long-term prognosis of the patients, we are following the HF patients in a cohort in the Tohoku area, ${ }^{15}$ which had been established 2.5 years before the Earthquake.

\section{Conclusions}

The Great East Japan Earthquake Disaster significantly in- creased the occurrence of CVDs, including HF, PTE and IE. Elderly patients with HF were significantly more affected by the Earthquake.

\section{Acknowledgments}

This work was supported by the contribution of the Japanese Circulation Society.

\section{Disclosures}

None.

\section{References}

1. Aoki T, Fukumoto Y, Yasuda S, Sakata Y, Ito K, Takahashi J, et al. The Great East Japan Earthquake Disaster and cardiovascular diseases. Eur Heart J 2012; 33: 2796-2803.

2. Watanabe H, Kodama M, Tanabe N, Nakamura Y, Nagai T, Sato M, et al. Impact of earthquakes on risk for pulmonary embolism. Int $J$ Cardiol 2008; 129: 152-154.

3. Watanabe H, Kodama M, Okura Y, Aizawa Y, Tanabe N, Chinushi M, et al. Impact of earthquakes on takotsubo cardiomyopathy. JAMA 2005; 294: 305-307.

4. Suzuki S, Sakamoto S, Miki T, Matsuo T. Hanshin-Awaji Earthquake and acute myocardial infarction. Lancet 1995; 345: 981.

5. Suzuki S, Sakamoto S, Koide M, Fujita H, Sakuramoto H, Kuroda T, et al. Hanshin-AwajiEearthquake as a trigger for acute myocardial infarction. Am Heart J 1997; 134: 974-977.

6. Leor J, Poole WK, Kloner RA. Sudden cardiac death triggered by an earthquake. $N$ Engl J Med 1996; 334: 413-419.

7. Zhang XQ, Chen M, Yang Q, Yan SD, Huang DJ. Effect of the wenchuan earthquake in china on hemodynamically unstable ventricular tachyarrhythmia in hospitalized patients. Am J Cardiol 2009; 103: 994-997.

8. Tsuchida M, Kawashiri MA, Teramoto R, Takata M, Sakata K, Omi $\mathrm{W}$, et al. Impact of severe earthquake on the occurrence of acute coronary syndrome and stroke in a rural area of Japan. Circ J 2009; 73: $1243-1247$.

9. Esler M, Kaye D. Sympathetic nervous system activation in essential hypertension, cardiac failure and psychosomatic heart disease. J Cardiovasc Pharmacol 2000; 35: S1-S7.

10. Segerstrom SC, Miller GE. Psychological stress and the human immune system: A meta-analytic study of 30 years of inquiry. Psychol Bull 2004; 130: 601-630.

11. Kario K. Disaster hypertension: Its characteristics, mechanism, and management. Circ J 2012; 76: 553-562.

12. Satoh M, Kikuya M, Ohkubo T, Imai Y. Acute and subacute effects of the Great East Japan Earthquake on home blood pressure values. Hypertension 2011; 58: e193-e194.

13. Nakano M, Kondo M, Wakayama Y, Kawana A, Hasebe Y, Shafee MA, et al. Increased incidence of tachyarrhythmias and heart failure hospitalization in patients with implanted cardiac devices after the Great East Japan Earthquake Disaster. Circ J 2012; 76: 1283-1285.

14. Gerin W, Chaplin W, Schwartz JE, Holland J, Alter R, Wheeler R, et al. Sustained blood pressure increase after an acute stressor: The effects of the 11 September 2001 attack on the New York City World Trade Center. J Hypertens 2005; 23: 279-284.

15. Shiba N, Nochioka K, Miura M, Kohno H, Shimokawa H, CHART2 Investigators. Trend of westernization of etiology and clinical characteristics of heart failure patients in Japan: First report from the Chart-2 Study. Circ J 2011; 75: 823-833. 